\title{
Quantitative Analysis of Cerebral Vessels in the Newborn Puppy: The Structure of Germinal Matrix Vessels May Predispose to Hemorrhage
}

\author{
BARBARA L. TROMMER, DENNIS R. GROOTHUIS, AND JOSEPH F. PASTERNAK \\ Departments of Pediatrics [B.L.T., J.F.P.] and Neurology [B.L.T., D.R.G., J.F.P.], Northwestern University \\ Medical School, Evanston Hospital, Evanston, Illinois 60201
}

\begin{abstract}
Intracerebral hemorrhage in premature infants commonly originates in the germinal matrix (GM). We performed a quantitative analysis of cerebral microvasculature from newborn puppies, a model for neonatal periventricular and intraventricular hemorrhage, at the light and electron microscopic level. GM vessels were compared with those of other brain regions in an effort to delineate pathogenetically significant structural features that might predispose to hemorrhage. Light microscopic examination revealed that GM vessel density (103.0 vessels $\left./ \mathrm{mm}^{2}\right)$ was similar to that in white matter $(98.3$ vessels/ $\left.\mathrm{mm}^{2}\right)$, but lower than that of cortex $\left(155.6\right.$ vessels $\left./ \mathrm{mm}^{2}\right)$ or caudate $\left(259.9\right.$ vessels $\left./ \mathrm{mm}^{2}\right)$. Mean blood vessel diameter was slightly larger in GM $(9.0 \mu)$ than cortex $(6.9 \mu)$, caudate $(7.9 \mu)$, and white matter $(8.9 \mu)$. Ultrastructurally, GM vessels were thinner along greater portions of their circumferences than vessels from other brain regions, as shown by their smaller ratio of vessel wall area/vessel lumen area and their greater fraction of vessel wall with thickness less than $0.25 \mu$. In addition, a significantly larger fraction of GM capillary wall lacked direct contact with perivascular structures. We postulate that the larger size, thinner walls, and diminished support from surrounding neuropil, which characterize GM vessels, may render them more susceptible to both physical (e.g. hypertension) and metabolic (e.g. hypoxia) insults than vessels from other brain regions. (Pediatr Res 22: 23-28, 1987)
\end{abstract}

\section{Abbreviations}

GM, germinal matrix

PIVH, periventricular and intraventricular hemorrhage

Although almost all PIVH in the premature neonate originates in the subependymal GM, the selective vulnerability of that region remains incompletely understood. Most studies of PIVH have focused on the physiologic aspects of its pathogenesis, with particular emphasis on blood flow. Previously, we demonstrated in the newborn beagle model for PIVH that blood flow to the GM is low relative to the rest of the brain (1). We have shown that autoregulation is intact in the GM of the beagle, although the insults associated with PIVH may operate beyond the autoregulatory range (2). Although GM blood flow increases with

Received October 3, 1986; accepted January 29, 1987

Correspondence and reprint requests Barbara L. Trommer, M.D., Division of Neurology Evanston Hospital, 2650 Ridge Avenue, Evanston, IL 60201

This work was supported by the Dee and Moody Research Fund (Evanston Hospital) and by NIH Grants KO4 NS00814, RO1 NS12745, PO1 NS20023 (DRG), and R23 NS20008 (JFP) systemic hypertension, the increase is not as great as it is in most cortical and subcortical structures (3). Therefore, the consistent localization of hemorrhage within the GM cannot be entirely explained by preferential shunting of blood flow to that region and must depend, in part, on other factors.

We studied structural properties of GM vasculature that might play a role in PIVH. Early work established a relationship between intraventricular and subependymal GM hemorrhage in the premature neonate (4) and the site of hemorrhage was identified as the capillary (5). A number of investigators have speculated that PIVH might result from increased vascularity (vessel density) of the GM as well as the presence of delicate poorly supported vessels thought characteristic of "immaturity" (4-9). We performed a quantitative comparison of GM microvasculature with that of other cerebral regions in which hemorrhage does not occur, in an attempt to delineate structural differences which might be important in the pathogenesis of PIVH. We studied puppies delivered at term because their germinal matrices are histologically comparable to those of human infants at the time of greatest risk for PIVH, and since they are subject to experimental hemorrhage (10).

\section{MATERIALS AND METHODS}

Specimen preparation. Brain specimens from two normal puppies less than $12 \mathrm{~h}$ of age were used for these studies. During ether anesthesia, the thorax was rapidly opened and the ascending aorta cannulated. The animal was killed and simultaneously fixed by initial perfusion with $1 \%$ glutaraldehyde- $0.8 \%$ paraformaldehyde in $0.2 \mathrm{M} \mathrm{NaCacodylate} \mathrm{buffer} \mathrm{(pH} \mathrm{7.2),} \mathrm{which} \mathrm{was}$ heparinized (1000 U/liter) and delivered at a controlled pressure of $50 \mathrm{~mm} \mathrm{Hg}$ until return to the right atrium was clear. The perfusion was continued with $5 \%$ glutaraldehyde- $4 \%$ paraformaldehyde until an approximate volume of $300 \mathrm{ml}$ had been infused (11). The brain was removed, sectioned coronally at 5 $\mathrm{mm}$ thickness, and fixed overnight in the stronger aldehyde solution.

Tissue samples $(3 \times 3 \times 1 \mathrm{~mm})$ were obtained by dissection from the following brain regions: 1) germinal matrix medial to the most rostral portion of head of caudate, 2) head of caudate, 3 ) white matter of the centrum semiovale, and 4) frontal cortex. The samples were postfixed in $2 \%$ osmium tetroxide, dehydrated in graded alcohols, and embedded in Araldite. One- $\mu$ sections were stained with toluidine blue. After appropriate regions were identified by light microscopy, thin sections for electron microscopy were cut, mounted on copper grids, and stained with uranyl acetate and lead citrate.

Light microscopic data analysis. To determine blood vessel density and size distribution, $1-\mu$ toluidine blue-stained sections were analyzed with a video-based digitizing system. Fields from each of the four brain regions were digitized at an initial magni- 
fication of $\times 320$, with a corresponding resolution of $4 \mu /$ pixel. The digitized images were displayed on a video monitor, and all vessels in each digitized image were traced using a digitizing tablet and interactive image processor. For each vessel, the least cross-sectional diameter, luminal area, location ( $\mathrm{x}$, y coordinates of the point in the center of the least cross-sectional diameter), and total area examined were stored by a computer. Data from individual sections of the same brain region were pooled for analysis. Vessel density was calculated from (number of vessels)/ (total area examined) and expressed as vessels $/ \mathrm{mm}^{2}$. Vessel size distribution (in terms of least cross-sectional diameter) was determined for 1) all vessels and 2) capillaries alone (in which capillaries were defined as vessels with least cross-sectional diameter $<10 \mu$ ). With this approach for preparation and analysis, capillaries stand out in well-fixed material (Fig. 1). In comparative studies in rat brain, using factor VIII/von Willebrand antibody with peroxidase-antiperoxidase staining and reticulin stains, we have determined that $>98 \%$ of the capillaries in a $1-\mu$ toluidine blue section are correctly identified (Groothuis DR, unpublished observations).

Electron microscopic data analysis. Electron micrographs of 200 vessels ( 61 from germinal matrix, 50 from cortex, 48 from caudate, and 41 from white matter) were obtained by photographing all vessels encountered with least cross-sectional diameter $<25 \mu$ with a Siemens 101 electron microscope at calibrated magnifications of either $\times 2400$ or $\times 4000$. Photographs were printed at a final magnification of $\times 7200$ or $\times 12,000$. Direct measurements were made from the electron micrographs to obtain 1) ratio of vessel wall area to luminal area, 2) wall thickness, and 3) percentage of capillary wall without supporting investment.

Vessel wall area was defined as the total area occupied in the electron micrograph by both endothelial and pericyte cytoplasm. Vessel wall area was determined by first obtaining the total area encompassed by the outer margin of either the endothelial cell or pericyte (when present), and then subtracting the luminal area and the area of any endothelial or pericyte nuclei. The area of these nuclei was subtracted to correct for the random placement of cell nuclei along the length of the vessels. The ratio of vessel wall area to luminal area was determined for each vessel.

Vessel wall thickness was determined by direct measurement from the electron micrographs. To express our data in a way that conveys "thinness" of the capillaries, we measured the fraction of each vessel wall circumference which had a wall thickness $<0.25 \mu$, and expressed the data as "fraction $<0.25 \mu$."

To express the degree to which each capillary was "supported" by extravascular structures, each vessel was examined to determine the fraction of the circumference in which potentially supporting structures were not in direct contact with the vessel basement membrane. This was expressed as "fraction bare." Although the presence or absence of direct contact between supporting structures and the vessel basement membrane was a qualitative decision, it was in most instances unambiguous (Fig. 2).

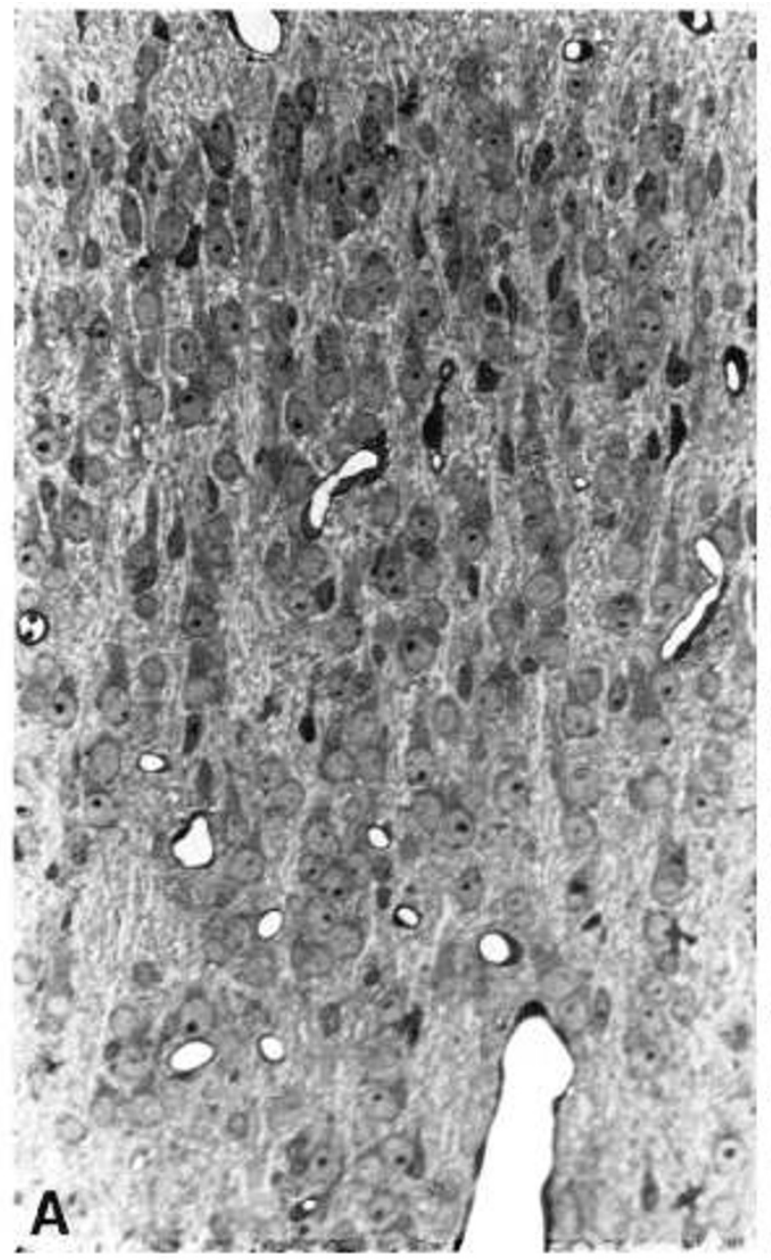

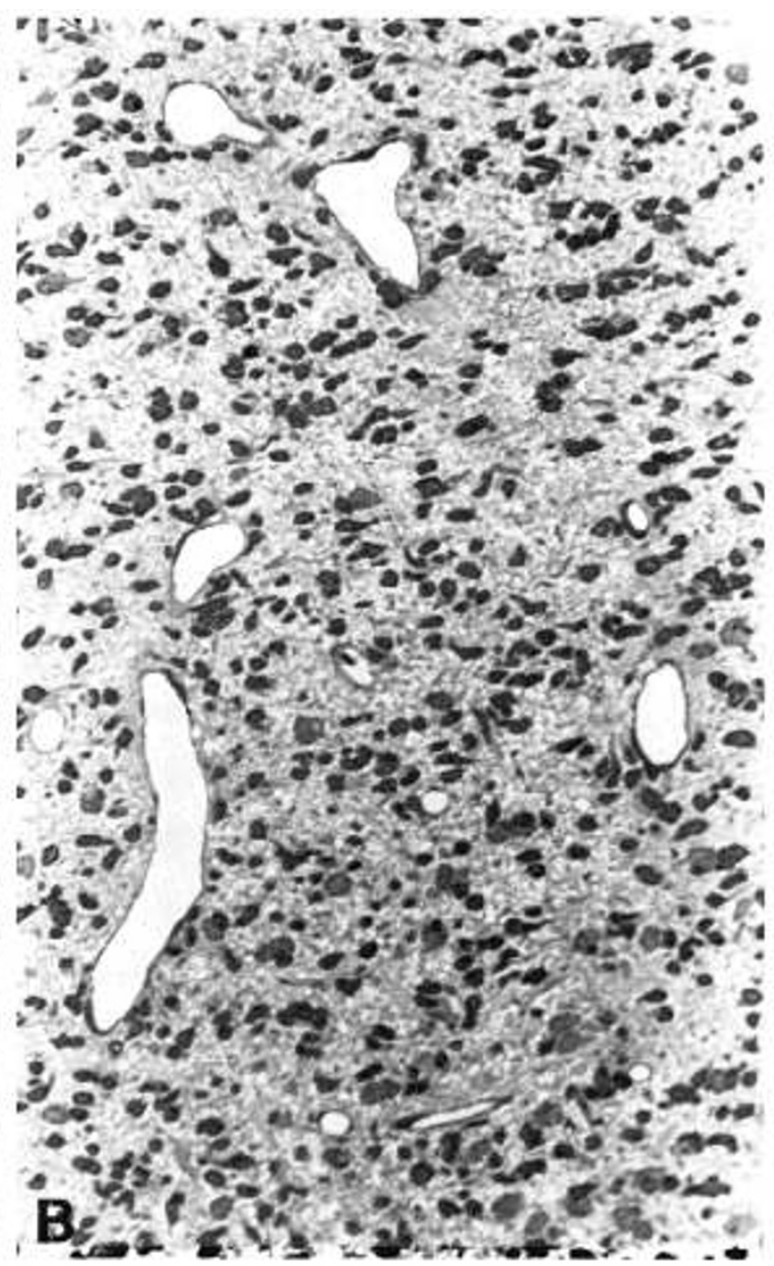

Fig. 1. Difference in the appearance of the vasculature between cerebral cortex $(A)$ and $\mathrm{GM}(B)$. Both photomicrographs were obtained from the same animal and represent perfusion-fixed material, cut at $1-\mu$ thickness and stained with toluidine blue. These low power light micrographs illustrate the higher incidence of vessels with a diameter $>9 \mu$ in the GM as well as the lower density of all blood vessels in the GM as compared to cerebral cortex. In addition, even in this light level photomicrograph, the capillary walls appear thinner and their walls less invested with supporting structures in the GM than in the cerebral cortex; magnification, $\times 40$. 

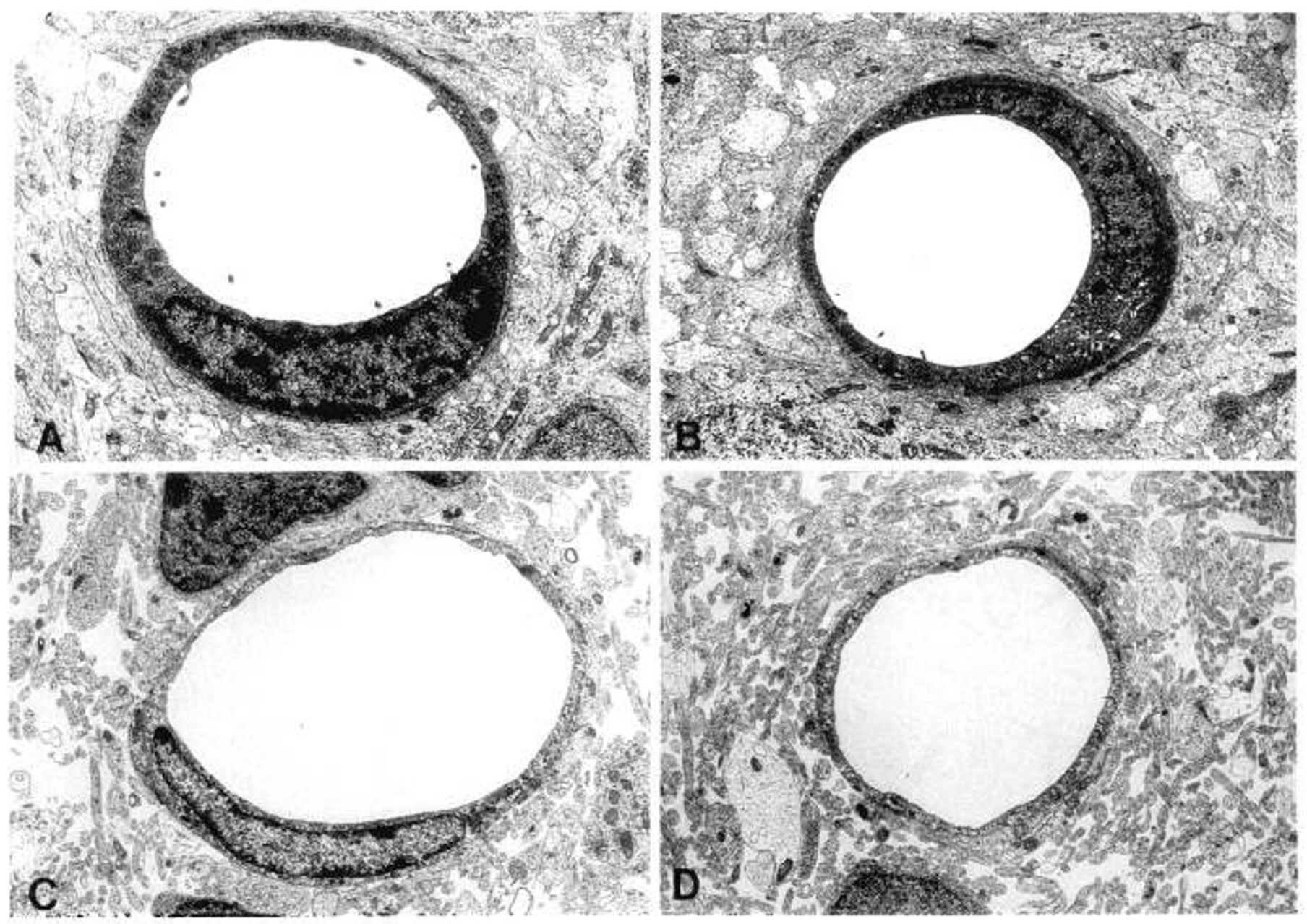

Fig. 2. Typical electron microscopic appearance of capillaries from the four different brain regions examined: cortex $(A)$, caudate $(B)$, germinal matrix $(C)$, and white matter $(D)$. The capillaries from the cortex and caudate are characterized by thicker walls relative to lumen size and compact processes surrounding the capillary. In contrast, the capillary from the GM has a thinner wall and the surrounding neuropil leaves much of the capillary wall bare. Magnification, $\times 12,000$.

Measurements of vessel size distribution and relative vessel wall area were compared using analysis of variance since we were comparing the means among four groups (the four brain regions) and since inspection of the data suggested normal distribution. The fraction of wall perimeter $<0.25 \mu$ and the fraction bare were analyzed using the order statistic since inspection of the data suggested they were not normally distributed (12).

\section{RESULTS}

Vessel density. The values of vessel density from each of the four different brain regions examined are shown in Table 1. Oneway analysis of variance showed that the vessel density was not the same for all groups $(p<0.001,3 \mathrm{df})$. Post hoc tests (Tukey HSD at $p<0.01$ ) showed that vessel density in GM was significantly less than that of caudate but not significantly different from that of cortex or white matter. Since a wide range of vessel density in cortex ( 93.4 to 195.0 vessels $/ \mathrm{mm}^{2}$ ) was seen within one animal, it is probable that different cortical layers were examined in different fields.

Vessel size distribution. The values of least cross-sectional diameter for all vessels, and for capillaries alone, are presented in Table 1 . For all vessels and capillaries alone, the germinal matrix vessels were larger than those from other brain regions. This difference was significant for cortex vessels (one-way analysis of variance, Tukey HSD at $p<0.001$ ) but not for those of caudate or white matter.
Electron microscopy. The electron microscopic appearance of vessels from all regions was similar to previously published descriptions in other species (13). The most notable regional differences concerned the basal lamina and the density of packing in the neuropil. The basement membrane was dense and continuous in vessels from the cortex and caudate; it was wispy and often discontinuous in germinal matrix. Basement membrane from white matter was intermediate in its characteristics. The size of the perivascular space was visibly increased in both GM and white matter in comparison to cortex and caudate, which corresponds to a general increased density of the neuropil in cortex and caudate and a lack of myelinated fibers in GM and white matter (Figure 2).

The ratios of wall area to luminal area are illustrated in Figure 3. One-way analysis of variance showed that the groups were different $(p<0.001,3 \mathrm{df})$. Post hoc tests showed that the difference was significant between $\mathrm{GM}(0.3 \pm 0.02$, mean $\pm \mathrm{SE})$ and cortex vessels $(0.49 \pm 0.04)(p<0.001$, Tukey HSD) and between GM and caudate vessels $(0.47 \pm 0.04)(p<0.01$, Tukey HSD), although not between GM and white matter vessels ( 0.41 $\pm 0.03)(p>0.05)$. This corresponded to a larger fraction of GM capillary wall with thickness $<0.25 \mu$ (Fig. 3). The fraction of GM capillary wall $<0.25 \mu(0.28 \pm 0.04$, mean \pm SE) was significantly larger than that for cortex $(0.11 \pm 0.02)$, caudate $(0.03 \pm 0.01)$, or white matter $(0.10 \pm 0.01)(p<0.05$, order statistic).

As is apparent in the electron micrographs of the pericapillary 
Table 1. Total area studied $\left(\mathrm{mm}^{2}\right)$, vessel density, and least diameter for each of the four regions that were examined

\begin{tabular}{|c|c|c|c|c|c|c|c|}
\hline \multirow[b]{2}{*}{ Brain region } & \multirow[b]{2}{*}{$\begin{array}{l}\text { Total } \\
\text { area } \\
\text { studied } \\
\left(\mathrm{mm}^{2}\right)\end{array}$} & \multicolumn{4}{|c|}{ All blood vessels } & \multicolumn{2}{|c|}{ Capillaries } \\
\hline & & $\mathrm{N}^{*}$ & $\begin{array}{l}\text { Density } \\
\mathrm{N} / \mathrm{mm}^{2}\end{array}$ & $\mathrm{~N} \dagger$ & $\begin{array}{c}\text { Least } \\
\text { diameter } \\
\mu \\
\end{array}$ & $\mathrm{N} \dagger$ & $\begin{array}{c}\text { Least } \\
\text { diameter } \\
\mu\end{array}$ \\
\hline GM & 1.702 & 4 & $102.3 \pm 7.6$ & 176 & $9.0 \pm 0.4$ & 138 & $6.7 \pm 0.1$ \\
\hline Cortex & 1.446 & 2 & $155.6 \pm 38.9$ & 225 & $6.0 \pm 0.2 \dagger$ & 210 & $5.6 \pm 0.1 \dagger$ \\
\hline Caudate & 0.376 & 3 & $259.9 \pm 3.6 \ddagger$ & 97 & $8.0 \pm 0.5$ & 81 & $6.5 \pm 0.2$ \\
\hline White matter & 1.160 & 3 & $98.3 \pm 11.8$ & 113 & $8.4 \pm 0.5$ & 88 & $6.3 \pm 0.2$ \\
\hline
\end{tabular}

* Number of fields that were examined.

$\dagger$ Number of vessels examined. "All blood vessels" refers to all vessels within an examined area; "capillaries" refers to all vessels with a least diameter $<10 \mu$. Values are means $\pm \mathrm{SE}$.

$\dagger$ Difference from GM value is significant at $p<0.001$ level.

$\ddagger$ Difference from GM is significant at $p<0$.01 level (analysis of variance, Tukey HSD).

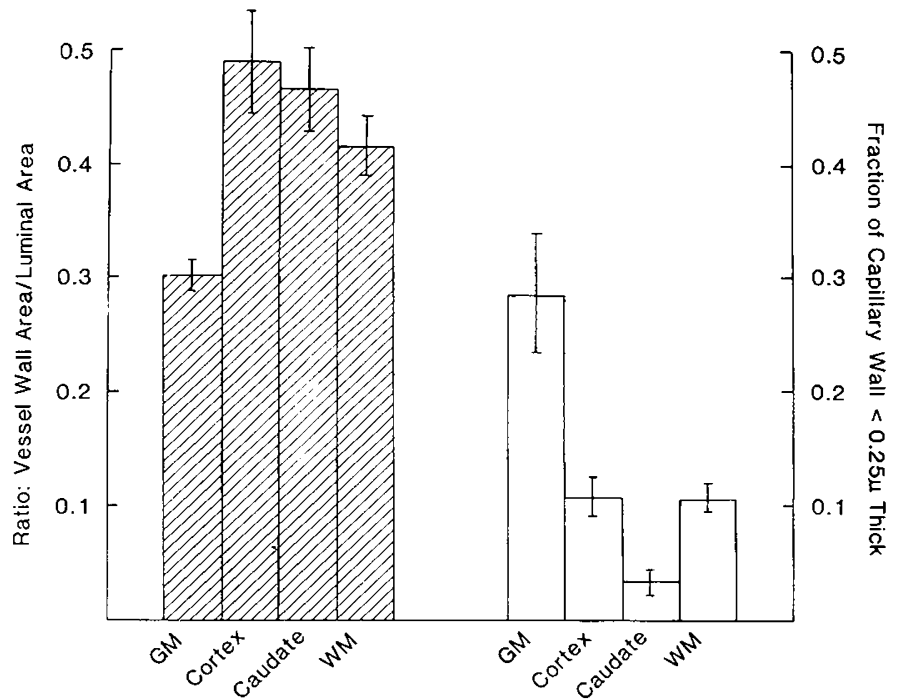

Fig. 3. The cross-hatched columns on the left show the ratio of the vessel wall area to the lumen area determined from measurements obtained in cross-sections of electron micrographs. The wall area from the GM vessels represents a smaller fraction of the luminal area than in vessels from the other brain regions. These differences were significant for cortex vessels $(p<0.001)$ and caudate vessels $(p<0.01)$ (analysis of variance, Tukey HSD). On the right is shown the fraction of capillary wall with a thickness $<0.25 \mu$. In this instance, the GM vessels have a significantly larger fraction of capillary wall $<0.25 \mu$ than vessels from any of the other brain regions ( $p<0.05$, order statistic). All values are mean $\pm \mathrm{SE}$.

environments (Fig. 2), a larger fraction of the basement membrane of the GM capillaries was not in contact with perivascular supporting elements (Table 2). For GM capillaries, a fraction of $0.12 \pm 0.012$ (mean $\pm \mathrm{SE}$ ) of the basement membrane was free from contact, which was significantly higher than the ratio for cortex $(0.02 \pm 0.004)$, caudate $(0.02 \pm 0.032)(p<0.001$, order statistic), or white matter vessels $(0.06 \pm 0.013)(0.01<p<0.05$, order statistic).

\section{DISCUSSION}

Our objective was to study the capillaries of the GM of the newborn beagle puppy in order to determine whether there were morphometric differences between these capillaries and capillaries from other brain regions. Our studies permit several observations. First, the mean value of GM vessel density was similar to that of white matter, but lower than that of cortex or caudate (Table 1). Second, mean vessel diameter was larger in GM than in other brain regions although this difference was significant only for cortex vessels (Table 1). Third, the ratio of vessel wall
Table 2. Number of vessels examined $(N)$ by electron microscopy in each of four different brain regions, and the "fraction bare," i.e. fraction of vessel perimeter which lacks direct contact or investment by perivascular supporting elements*

\begin{tabular}{lll}
\hline Brain region & $\mathrm{N}$ & Fraction bare \\
\hline GM & 61 & $0.12 \pm 0.012$ \\
Cortex & 50 & $0.02 \pm 0.004$ \\
Caudate & 48 & $0.02 \pm 0.032$ \\
White matter & 41 & $0.06 \pm 0.013$ \\
\hline
\end{tabular}

* Values are mean \pm SE. A significantly larger fraction of GM capillary wall lacks direct contact with perivascular structures than vessels from any of the other three brain regions ( $p<0.05$, order statistic).

area/vessel lumen area was greater in capillaries of other brain regions than in GM capillaries. This difference was significant for cortex and caudate vessels (Fig. 3). Fourth, the fraction of blood vessel wall with a thickness $<0.25 \mu$ was significantly greater in GM capillaries than in capillaries from the other brain regions (Fig. 3). These latter two observations imply that a larger part of the circumference of GM capillaries is thinner than that of capillaries from other brain regions. Fifth, two to five times as much germinal matrix capillary basement membrane lacked direct contact with surrounding neuropil structures (Table 2). This observation supports the concept that GM capillaries have less structural support from surrounding structures than other brain capillaries.

Few studies are available for direct comparison with our data. Lenn and Whitmore (13) reported endothelial to lumen volume ratios of $0.19-0.59$ in germinal matrix capillaries of rhesus monkey fetuses in the 3rd trimester of gestation. This corresponds to our vessel wall area/vessel lumen area of 0.3 . Nelson et al. (14) found the ratio of endothelial to lumen area in newborn beagle puppy GM vessels to be smaller than that of watershed cortical vessels, but consistently found values greater than one (14). Our vessels and those of Lenn and Whitmore (13) appeared fully expanded with smoothly contoured luminal surfaces (Fig. 2) in contrast to the marked infolding evident in the typical vessel of Nelson et al. (14). This discrepancy may reflect differences in perfusion pressure or other aspects of fixation, and illustrates the need for caution in the interpretation of the absolute values of morphometric data from fixed specimens. It is known, for example, that differential effects of perfusion fixation may result from variations in vascular supply and vessel permeability (15). In our work in particular, the lack of support from surrounding tissue may have allowed the GM vessels to expand more fully than the other vessels during perfusion. We hoped to minimize this effect by choosing a perfusion pressure in the physiologic range for newborn puppies (2).

We do not agree with Lenn and Whitmore (13) that the apparent low tissue support for GM vessels (Table 2 ) is likely to 
result from fixation artifact. It is known, as they suggest, that in the absence of fenestrated capillaries fixation with conventional aldehyde solutions may lead to osmotically induced swelling of neurons and glia (15). However, if this were the case, underestimation rather than exaggeration of the extracellular space would be expected (Fig. 2). The possible differential effects of fixatives on neonatal brain tissue remain to be explored in more detail.

The diameters of "all vessels" and of capillaries in GM in our study were larger than those from any other brain region (Table 1). However, in addition, the mean capillary diameters in cortex, caudate, and white matter were slightly larger than values reported from fixed material in adult animals $(16,17)$. Since the mean diameter of capillaries in mature rat cortex when measured with our analyzing system (Groothuis DR, unpublished observations) corresponds with values reported by others, measurements made with our system are validated. Therefore, the large mean capillary diameters in Table 1 suggest a general difference in the neonatal puppy brain and not one confined to GM alone. We are unaware of other measurements of capillary diameter in newborn puppies with which we can compare our data.

Fung (18), working with systemic capillaries, reasoned that capillaries appearing rigid (e.g. those embedded in mesentery or muscle) receive support from surrounding tissue-like "tunnels in a gel." In contrast, when the surrounding tissue is small in size and insignificant in effect (e.g. pulmonary capillaries surrounded by air), wall tension determines compliance with respect to transmural pressure. We suggest that the ability of GM vessels to withstand increases in transmural pressure may depend on wall tension to a greater extent than might be expected for the better supported vessels in other brain regions. Furthermore, GM vessels may be subject to higher wall tension than other cerebral vessels because of their larger size and thinner walls. Together, these properties may make GM vessels less able to withstand increases in pressure and more prone to rupture.

It is most likely that both structural and physiologic factors contribute to the pathogenesis of PIVH. Lenn and Whitmore (13) hypothesized that to explain the predisposition to PIVH in premature infants, pathogenetically important morphologic features of GM should be present in the 3rd trimester and rapidly change near term. Since they failed to demonstrate such changes in normal rhesus monkey fetuses, they concluded that their findings "do not support a structural immaturity or specialization of the germinal matrix predisposing to germinal matrix hemorrhage" and recommended emphasis on "physiologic parameters" instead. The apparent lack of support for structural specialization in their data may reflect their choice of an animal model in which experimental hemorrhage has not been produced. More importantly, we would argue that the absence of rapid morphologic changes in GM near term (i.e. as the incidence of PIVH declines) should not deflect attention from the role of morphologic features present at the time of predilection for PIVH. Rather, we believe that the selective vulnerability of GM relates to a time window during which two developmental processes, one physiologic and one structural, overlap. As term approaches, each becomes less important as the interaction of the two processes diminishes.

Our study was performed in term newborn puppies, a time chosen to reflect the chronologic constraints of experimental hemorrhage. We were able to demonstrate that GM vessels are distinguished structurally from vessels in hemorrhage-resistant areas by their lack of relative wall thickness and support from basal lamina and surrounding neuropil. In similarly timed experiments on littermate puppies, we demonstrated that the rostral GM is a physiologically active region. Regional cerebral blood flow is low at baseline and increases disproportionately with systemic hypertension (1-3). Although autoregulation is intact, mean arterial pressure $(65 \mathrm{~mm} \mathrm{Hg})$ is only slightly below the upper limit of the autoregulatory plateau $(70-75 \mathrm{~mm} \mathrm{Hg}$ ), and elevations of mean arterial pressure above $80 \mathrm{~mm} \mathrm{Hg}$ are not uncommon during handling (2). Furthermore, we have demon- strated an increase in local cerebral glucose utilization in this region following systemic hypotension or hypoxemia $(21,22)$. Glucose hypermetabolism is both a marker for local brain injury (23-25) and a source for accumulation of potentially vasotoxic lactate and hydrogen ions.

The structural and physiologic features of GM involution and the tempo at which they progress are not yet fully known. It is probably not useful to discuss "maturity" in a brain region destined to involute, but with time GM vessels seem to acquire some of the structural properties of hemorrhage-resistant areas. For example, maturational studies in human fetal GM through 26 wk gestation $(19,20)$ have shown a trend toward expansion of glial investment with concomitant reduction in the extracellular space, and an increase in the definition, continuity, and thickness of basal lamina. Since the involution of GM is known to progress from caudal portions forward, we may speculate from static data that blood flow, reactivity of blood flow, and energy metabolism progressively decrease in rostral GM as involution proceeds. Furthermore, late in gestation cerebral blood flow shifts away from brainstem, basal ganglia, and periventricular structures to a predominantly cortex-oriented circulation (6). Thus, late in gestation it is likely that neither structural nor physiologic features of GM would predispose it to hemorrhage. However, at a particular time in development (as demonstrated in the term puppy), both thin-walled, poorly supported vessels and reactivity of blood flow and glucose utilization are present in rostral GM. In our view, it is this coincidence of physiologic responsiveness and structural fragility that renders rostral GM particularly susceptible to hemorrhage.

Acknowledgment. Presented in abstract form at the Annual Meeting of the Child Neurology Society, Williamsburg, VA, October 13, 1983.

\section{REFERENCES}

1. Pasternak JF, Groothuis DR, Fischer JM, Fischer DP 1982 Regional cerebral blood flow in the newborn beagle pup: the germinal matrix is a "low-flow" structure. Pediatr Res 16:499-503

2. Pasternak JF, Groothuis DR 1985 Autoregulation of cerebral blood flow in the newborn beagle puppy. Biol Neonate 48:100-109

3. Pasternak JF, Groothuis DR, Fischer JM, Fischer DP 1983 Regional cerebral blood flow in the beagle model of neonatal intraventricular hemorrhage: studies during systemic hypertension. Neurology 33:559-566

4. Leech RW, Kohnen P 1974 Subependymal and intraventricular hemorrhages in the newborn. Am J Pathol 77:465-475

5. Hambleton G. Wigglesworth JS 1976 Origin of intraventricular hemorrhage in the preterm infant. Arch Dis Child 51:651-659

6. Pape KE, Wigglesworth JJ 1975 Hemorrhage, Ischemia, and the Perinatal Brain. JB Lippincott, Philadelphia, pp 143-145

7. Milligan DWA 1980 Failure of autoregulation and intraventricular hemorrhage in preterm infants. Lancet 1:896-898

8. Gruenwald P 1951 Subependymal cerebral hemorrhage in premature infants and its relation to various injurious influences at birth. Am J Obstet Gynecol 61:1285-1292

9. Ross JJ, Dimette RM 1965 Subependymal cerebral hemorrhage in infancy. Am J Dis Child 1 10:531-542

10. Goddard J, Lewis RM, Alcala H, Zeller RS 1980 Intraventricular hemorrhage-an animal model. Biol Neonate 37:39-52

11. Karnovsky MJ 1965 A formaldehyde-glutaraldehyde fixative of high osmolarity for use in electron microscopy. J Cell Biol 27:270-278

12. Snedecor GW, Cochran WG 1980 Statistical Methods. Iowa State University Press, Ames, IA, pp 135-148

13. Lenn NJ, Whitmore L 1985 Gestational changes in the germinal matrix of the normal rhesus monkey fetus. Pediatr Res 19:130-135

14. Nelson RM, Leuschen MP, Shuman RM 1982 A morphometric analysis of germinal matrix and cortical capillaries in beagle pups. Perinatal Intracranial Hemorrhage Conference, Washington, DC, Syllabus 6-8, Ross Laboratories. Columbus, $\mathrm{OH}$

15. Hayat MA 1981 Fixation for Electron Microscopy. Academic Press. New York, pp 265-269

16. Hunziker O, Frey H, Schulz U 1974 Morphometric investigations of capillaries in the brain cortex of the cat. Brain Res $65: 1-11$

17. Pawlik G, Rackl A, Bing RJ 1981 Quantitative capillary topography and blood flow in the cerebral cortex of cats: an in vivo microscopic study. Brain Res 208:35-58

18. Fung YC 1980 Structural mechanics of microvasculature. In: Gross JF, Popel A (eds) Mathematics of Microcirculation Phenomena. Raven Press. New York, pp $1-16$ 
19. Povlishok JT, Martinez AJ, Moossy J 1977 The fine structure of blood vessels of the telencephalic germinal matrix in the human fetus. Am J Anat 149:439452

20. Larroche JC 1982 The fine structure of matrix capillaries in human embryos and young fetuses. Perinatal Intracranial Hemorrhage Conference, Washington, DC, Syllabus 2-5, Ross Laboratories, Columbus, $\mathrm{OH}$

21. Pasternak JF, Groothuis DR 1983 Systemic hypotension causes parasagittal and periventricular hypermetabolism in conscious newborn puppies. Ann Neurol 14:357-358

22. Pasternak JF, Schlageter K, Hayden R 1984 The effects of hypoxemia and hypotension on local cerebral glucose utilization in the awake newborn puppy. Pediatr Res 18(part 2):333A

23. Welsh FA, Greenberg JH, Jones SC, Ginsberg MD, Reivich M 1980 Correlation between glucose utilization and metabolite levels during focal ischemia in cat brain. Stroke 11:79-84

24. Raichle ME 1983 The pathophysiology of brain ischemia. Ann Neurol 13:210

25. Ginsberg MD, Welsh FA, Budd WW 1980 Deleterious effect of glucose pretreatment on recovery from diffuse cerebral ischemia in the cat. I. Local cerebral blood flow and glucose utilization. Stroke 11:347-354 